\begin{tabular}{|c|l|}
\hline Title & $\begin{array}{l}\text { Double magnetic tunnel junctions with cross magnetization configurations for electrical detection of domain-wall } \\
\text { structures }\end{array}$ \\
\hline Author(s) & Uemura, Tetsuy a; Sawada, Keisuke; Matsuda, Ken-ichi; Y amamoto, Masafumi \\
\hline Citation & $\begin{array}{l}\text { A pplied Physics Letters, 95(1), 012502 } \\
\text { https://doi.org/10.1063/.3168514 }\end{array}$ \\
\hline Issue Date & 2009-07-06 \\
\hline Doc URL & http://hdl.handle.net/2115/38855 \\
\hline Rights & $\begin{array}{l}\text { Copyright 2009 A merican Institute of Physics. This article may be downloaded for personal use only. A ny other use } \\
\text { requires prior permission of the author and the A merican Institute of Physics. }\end{array}$ \\
\hline Type & article \\
\hline File Information & 95-1_012502.pdf \\
\hline
\end{tabular}

Instructions for use 


\title{
Double magnetic tunnel junctions with cross-magnetization configurations for electrical detection of domain-wall structures
}

\author{
Tetsuya Uemura (植村哲也), ${ }^{a}$ Keisuke Sawada（澤田圭佑), Ken-ichi Matsuda（松田健一), \\ and Masafumi Yamamoto (山本眞史) \\ Division of Electronics for Informatics, Hokkaido University, Sapporo 060-0814, Japan
}

(Received 27 May 2009; accepted 14 June 2009; published online 7 July 2009)

\begin{abstract}
A device consisting of double magnetic tunnel junctions with cross-magnetization configurations is proposed to enable simultaneous electrical detection of both the structure and motion of a domain-wall (DW). Operation of this device has been confirmed through micromagnetic simulation. Owing to the cross-magnetization configurations, two types of DW structure formed in a ferromagnetic wire were clearly identified: a transverse wall (TW) in which the magnetization at the center of the wall is directed transversely to the wire axis and a vortex wall (VW) in which the magnetization circulates in the plane around a small perpendicular vortex core. In addition to the structural difference between TW and VW, the velocity of the DW motion was detected through the time response of the tunneling magnetoresistance. () 2009 American Institute of Physics. [DOI: $10.1063 / 1.3168514]$
\end{abstract}

The structure and motion of domain-walls (DWs) formed in a nanoscale ferromagnetic wire have been studied intensively from various scientific and technological points of view. ${ }^{1-7}$ Recently, the electrical detection of DW motion using a single magnetic tunnel junction (MTJ) was reported. ${ }^{8}$ This method enabled detection of the position and velocity of the DW through the tunneling magnetoresistance (TMR) effect on a nanosecond time scale. However, it was difficult to identify the DW structure through this method. In general, two types of DW structure are formed in a ferromagnetic wire: a transverse wall (TW) in which the magnetization at the center of the wall is directed transverse to the wire axis and a vortex wall $(\mathrm{VW})$ in which the magnetization circulates in the plane about a small perpendicular vortex core (Fig. 1) ${ }^{6,7}$ It is important to identify the DW structure-i.e., whether it is TW or VW-because the properties of the DW motion, such as velocity, in the ferromagnetic wire strongly depend on the DW structure. In this paper, we propose a device consisting of double MTJs with cross-magnetization configurations, in which the magnetization of a free layer is oriented orthogonally to the magnetization of a pinned layer, for the purpose of simultaneous electrical detection of both the structure and motion of the DW. We have confirmed the operation of this device through micromagnetic simulation.

Figures 2(a) and 2(b) show the structure of the proposed device and its operation principle. A pair of MTJs is formed on a ferromagnetic wire, which is shared by the MTJs as their lower electrodes. In the conventional structure described in Ref. 8, a single MTJ whose pinned direction is parallel to the wire axis is formed on a ferromagnetic wire. Since the magnetization direction of the ferromagnetic wire, except for the DW region, lies along the wire axis because of shape anisotropy, the TMR value for the conventional structure can be described as ${ }^{8}$

\footnotetext{
${ }^{\text {a)} E l e c t r o n i c ~ m a i l: ~ u e m u r a @ i s t . h o k u d a i . a c . j p . ~}$
}

$$
R=\left(\frac{L_{\mathrm{P}}+L_{\mathrm{AP}}}{R_{\mathrm{P}} L_{\mathrm{P}}}+\frac{L_{\mathrm{P}}+L_{\mathrm{AP}}}{R_{\mathrm{AP}} L_{\mathrm{AP}}}\right)^{-1},
$$

where $R_{\mathrm{P}(\mathrm{AP})}$ is the TMR value for the parallel (antiparallel) configuration and $L_{\mathrm{P}(\mathrm{AP})}$ is the wire length of the part at which the parallel (antiparallel) magnetization configuration occurs. The TMR value therefore changes depending on the DW position in the wire. However, it is insensitive to a difference in the DW structure.

To make the TMR value structure-sensitive, we adopted a cross-magnetization configuration for the MTJ. This cross configuration enables us to identify whether the DW structure entering into the MTJ region is TW or VW through the TMR time response. Suppose the pinned direction of the upper electrode is fixed to the $y$-axis direction. Here, we set the $x$-axis and $y$-axis to the directions of the wire length and width, respectively (see Fig. 2). In the TW structure, the magnetization direction in the wall rotates to the $+y$ or $-y$ direction. We will hereafter refer to the TW structure in which the magnetization direction rotates to the $+y(-y)$ direction as $\mathrm{TW}_{\mathrm{U}(\mathrm{D})}$. When the $\mathrm{TW}_{\mathrm{U}(\mathrm{D})}$ enters the MTJ region, the TMR decreases (increases) because the magnetization component parallel (antiparallel) to the pinned direction in-

(a)

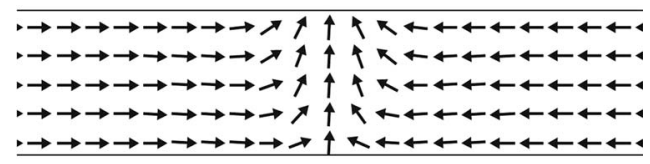

(b)

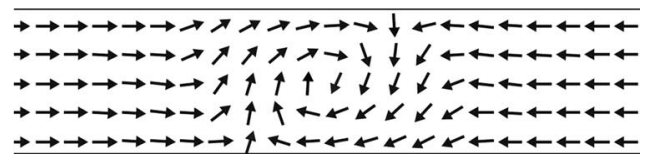

FIG. 1. DW structures stably formed in a ferromagnetic wire. (a) TW. (b) VW. 


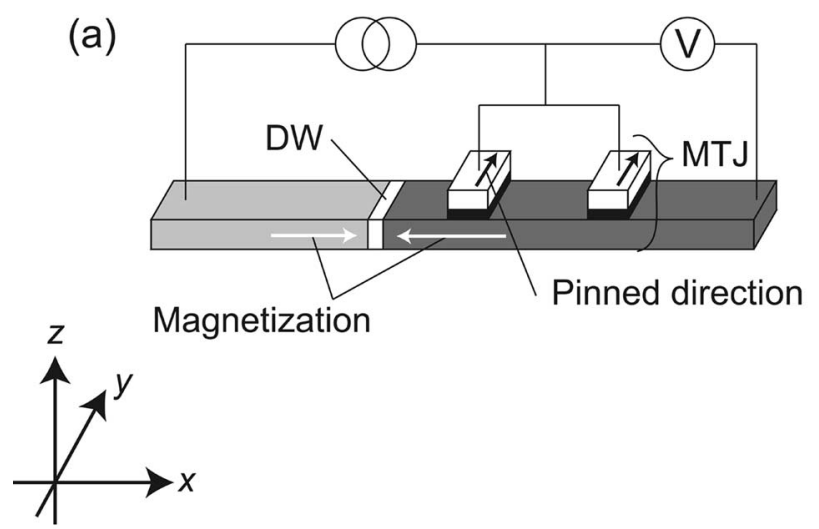

(b)

TW
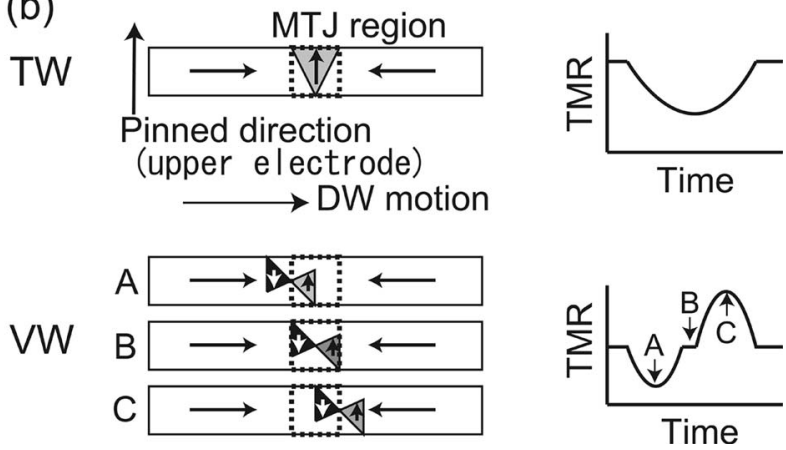

FIG. 2. (a) Proposed device structure consisting of double MTJs with crossmagnetization configurations used for the simultaneous electrical detection of both the structure and motion of a DW. (b) Operation principle of the proposed device.

creases. However, the $+y$ and $-y$ components in the VW structure are almost equal. We will refer to the VW structure in which the magnetization circulates clockwise (counterclockwise) in the $x y$-plane as $\mathrm{VW}_{\mathrm{CW}(\mathrm{CCW})}$. When the $\mathrm{VW}_{\mathrm{CW}(\mathrm{CCW})}$ is entering the MTJ region, the TMR value first increases (decreases) because the antiparallel (parallel) component increases. The TMR value then returns to almost its initial value when the $\mathrm{VW}_{\mathrm{CW}(\mathrm{CCW})}$ has completely entered the MTJ region because the parallel and antiparallel components become almost equal. Finally, the TMR decreases (increases) when the $\mathrm{VW}_{\mathrm{CW}(\mathrm{CCW})}$ is exiting the MTJ region because the antiparallel (parallel) component decreases. Thus, the TMR time traces for the TW motion differ from those for the VW motion, resulting in the DW structures being identified through these TMR response curves. Furthermore, the use of double MTJs enables the velocity of the DW motion to be detected through the time response because the same trace is repeated twice due to the double MTJs.

We calculated TMR values in this device when the DW driven by the magnetic field passed through the MTJ regions. We used half-metallic Heusler alloy $\mathrm{Co}_{2} \mathrm{MnSi}$ (CMS) ferromagnetic electrodes (both wire and upper electrodes) in the simulation based on an experimental demonstration of high TMR ratios of up to about $180 \%$ at room temperature (RT) for CMS/MgO/CMS MTJs. ${ }^{9-11}$ The length $(L)$ and width $(W)$ of the CMS wire were assumed to be $12 \mu \mathrm{m}$ and $250 \mathrm{~nm}$, respectively, and the thickness $(t)$ was 2.5 or $10 \mathrm{~nm}$. Each MTJ formed on the CMS wire was $250 \times 250 \mathrm{~nm}^{2}$ and the space between them was $800 \mathrm{~nm}$.

To calculate the TMR values, we divided the CMS wire into rectangular cells that were $2.5 \times 2.5 \times t \mathrm{~nm}^{3}$, and the

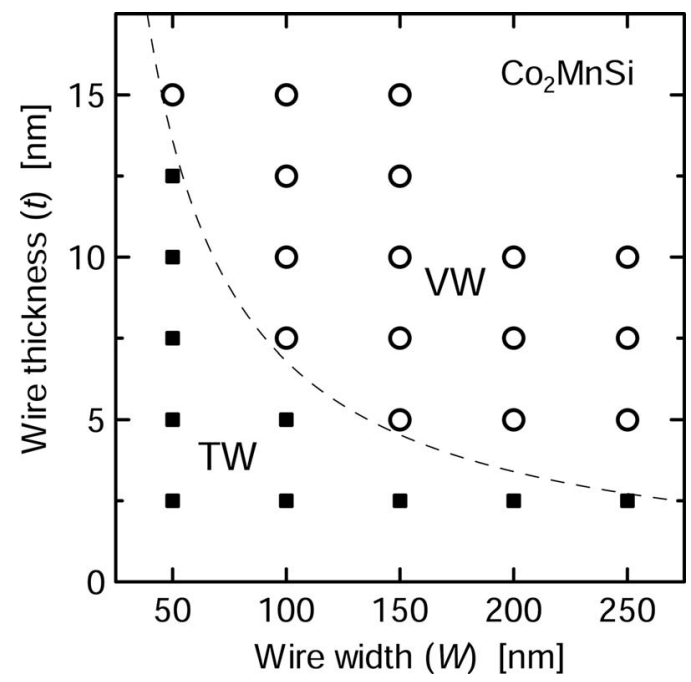

FIG. 3. Energetically favorable DW structures formed in the CMS wire as functions of wire width $(W)$ and thickness $(t)$.

magnetization direction in each rectangular cell was simulated by solving the Landau-Lifshitz-Gilbert equation. ${ }^{12}$ The magnetization was almost uniform inside each cell because the lateral cell size was smaller than the exchange length defined by $\sqrt{2 A / \mu_{0} M_{S}^{2}}$, where $A$ is the exchange stiffness constant and $M_{S}$ is a saturation magnetization. Unlike conventional MTJs, the angle of the magnetization of the $i$ th cell with respect to the pinned direction $\theta_{i}$ takes an arbitrary value. Therefore, we used the extended Julliere model given by ${ }^{13,14}$

$$
R_{i}=R_{0}\left[1+\frac{P^{2}}{1+P^{2} \cos \theta_{i}}\left(1-\cos \theta_{i}\right)\right]
$$

to calculate the TMR value for the $i$ th cell, $R_{i}$, where $P$ is the spin polarization of the CMS and $R_{0}$ is the TMR value when both magnetization vectors are parallel. The total TMR value was calculated as parallel connections of $R_{i}$ for the cells involved in the MTJ region.

The material parameters of the CMS used in the simulation were as follows: magnetization of $M_{S}=852 \times 10^{3} \mathrm{~A} / \mathrm{m}$, cubic magnetocrystalline anisotropy constant of $K_{1}=7.0$ $\times 10^{5} \mathrm{~J} / \mathrm{m}^{3}$, exchange stiffness constant of $A=4.6$ $\times 10^{-12} \mathrm{~J} / \mathrm{m},{ }^{15}$ damping constant of $\alpha=0.003,{ }^{16}$ and effective spin polarization of $P=0.687$. The value of $P$ was estimated from the TMR ratio of $179 \%$, which we recently demonstrated experimentally in $\mathrm{CMS} / \mathrm{MgO} / \mathrm{CMS}$ MTJs at room temperature. We simulated the motion of four kinds of DW structure: $\mathrm{TW}_{\mathrm{U}}, \mathrm{TW}_{\mathrm{D}}, \mathrm{VW}_{\mathrm{CW}}$, and $\mathrm{VW}_{\mathrm{CCW}}$. Magnetic fields of 1 and 5 Oe, respectively, were applied along the wire direction to drive the TW and VW.

Figure 3 indicates energetically favorable DW structures as a function of the geometry $(t$ and $W$ ) of the CMS wire. We simulated the time evolution of the energy of both the TW and VW for each $W$ and $t$ to determine the energetically favorable DW structure. The simulation results showed that the energetically favorable DW structure changed from the $\mathrm{TW}$ to the VW as either $W$ or $t$ was increased. The boundary curve between TW and VW was given by $t \times W=C$, where the constant value of $C$ was approximately $680 \mathrm{~nm}^{2}$. Similar results were reported for $\mathrm{NiFe}$ wires, where the value of $C$ was approximately $1500 \mathrm{~nm}^{2}{ }^{6,17}$ The smaller value of $C$ for the CMS wire compared to that for NiFe is possibly due to 
(a) TWU

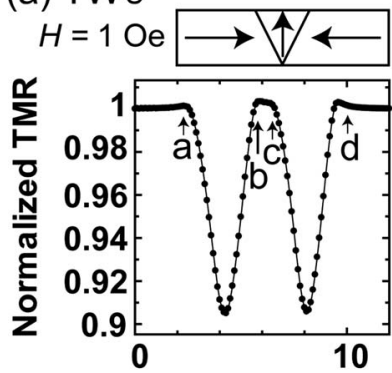

(c) $\mathrm{VWcW}$

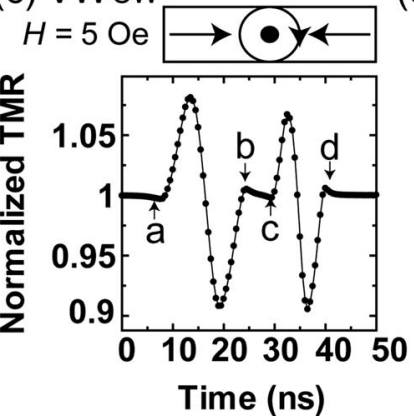

(b) TWD

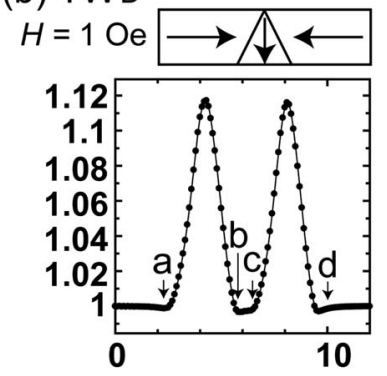

(d) VWccw

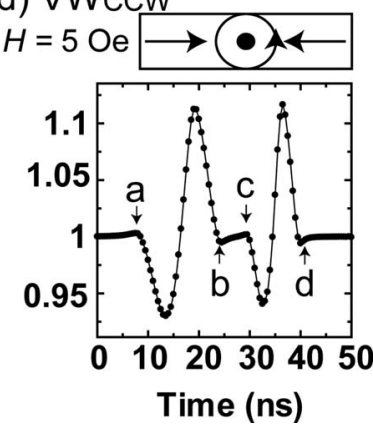

FIG. 4. Time traces of the normalized TMR values for the DW motion of (a) $\mathrm{TW}_{\mathrm{U}}$, (b) $\mathrm{TW}_{\mathrm{D}}$, (c) $\mathrm{VW}_{\mathrm{CW}}$, and (d) $\mathrm{VW}_{\mathrm{CCW}}$. Points A (c) and $\mathrm{B}$ (d) respectively indicate the time at which the DW began to enter the first (second) MTJ and finished passing through the first (second) MTJ.

the smaller exchange length ${ }^{6}$ in the CMS. Further studies on the stability of the DW structures are, however, beyond the scope of this paper. Because the TW and VW were stable at $(W, t)=(250,2.5)$ and $(250,10)$, respectively, we used CMS wire with $(W, t)=(250,2.5)$ for the simulation of TW motion and that with $(W, t)=(250,10)$ for the simulation of VW motion.

Figure 4 shows time traces of the TMR values for the DW motion of (a) $\mathrm{TW}_{\mathrm{U}}$, (b) $\mathrm{TW}_{\mathrm{D}}$, (c) $\mathrm{VW}_{\mathrm{CW}}$, and (d) $\mathrm{VW}_{\mathrm{CCW}}$. The TMR values were normalized by the TMR at the time when the DW was outside the MTJ regions. Points A (C) and B (D) in the figure respectively indicate the time at which the DW began to enter the first (second) MTJ and finished passing through the first (second) MTJ. As shown, we obtained four TMR traces that differed depending on the DW structures. The most significant difference in the TMR traces between the TW and VW is that the TW produced a

single peak structure when it passed through each junction, while the VW produced a peak and valley structure. These TMR traces can be used to identify the DW structure. Furthermore, the velocity of the DW motion could be estimated from the time difference between B and C. We found that the TW structure moved faster than the VW structure.

In conclusion, we have proposed a device consisting of double MTJs with cross-magnetization configurations, which can be used for simultaneous electrical detection of both the structure and motion of a DW. Micromagnetic simulation clearly demonstrated that the DW structure could be identified as either TW or VW, with simultaneous detection of the velocity of DW motion, through the TMR time response of the MTJs.

This work was supported in part by Grants-in-Aid for Scientific Research (Grant Nos. 20246054 and 21360140) and a Grant-in-Aid for Scientific Research on Priority Area "Creation and control of spin current" (Grant No. 19048001) from the MEXT, Japan.

${ }^{1}$ S. S. P. Parkin, M. Hayashi, and L. Thomas, Science 320, 190 (2008).

${ }^{2}$ K. Yamada, S. Kasai, Y. Nakatani, K. Kobayashi, H. Kohno, A. Thiaville, and T. Ono, Nature Mater. 6, 269 (2007).

${ }^{3}$ J.-Y. Lee, K.-S. Lee, S. Choi, K. Y. Guslienko, and S.-K. Kim, Phys. Rev. B 76, 184408 (2007).

${ }^{4}$ D. A. Allwood, G. Xiong, C. C. Faulkner, D. Atkinson, D. Petit, and R. P. Cowburn, Science 309, 1688 (2005).

${ }^{5}$ N. Ohshima, H. Numata, T. Suzuki, S. Fukami, K. Nagahara, and N. Ishiwata, J. Appl. Phys. 103, 07D914 (2008).

${ }^{6}$ R. D. McMichael and M. J. Donahue, IEEE Trans. Magn. 33, 4167 (1997).

${ }^{7}$ M. Kläui, J. Phys.: Condens. Matter 20, 313001 (2008).

${ }^{8}$ K. Kondou, N. Ohshima, S. Kasai, Y. Nakatani, and T. Ono, Appl. Phys. Express 1, 061302 (2008).

${ }^{9}$ Available online at http://math.nist.gov/oommf/.

${ }^{10}$ T. Ishikawa, S. Hakamata, K.-i. Matsuda, T. Uemura, and M. Yamamoto, J. Appl. Phys. 103, 07A919 (2008).

${ }^{11}$ T. Ishikawa, N. Itabashi, T. Taira, K.-i. Matsuda, T. Uemura, and M. Yamamoto, Appl. Phys. Lett. 94, 092503 (2009).

${ }^{12}$ T. Ishikawa, N. Itabashi, T. Taira, K.-i. Matsuda, T. Uemura, and M. Yamamoto, J. Appl. Phys. 105, 07B110 (2009).

${ }^{13}$ M. Jullière, Phys. Lett. 54A, 225 (1975).

${ }^{14}$ Y. Higo, H. Shimizu, and M. Tanaka, J. Appl. Phys. 89, 6745 (2001).

${ }^{15}$ O. Gaier, J. Hamrle, S. J. Hermsdoerfer, H. Schultheiß, B. Hillebrands, Y. Sakuraba, M. Oogane, and Y. Ando, J. Appl. Phys. 103, 103910 (2008).

${ }^{16}$ R. Yilgin, Y. Sakuraba, M. Oogane, S. Mizukami, Y. Ando, and T. Miyazaki, Jpn. J. Appl. Phys., Part 2 46, L205 (2007).

${ }^{17}$ Y. Nakatani, A. Thiaville, and J. Miltat, J. Magn. Magn. Mater. 290, 750 (2005). 